the peculiar position in which he stands." Clinical scientists could well use this maxim as their guide in constructing the best clinical trial for the condition they wish to study.

${ }^{1}$ Jones, F E, Biomedicine, 1978, 28, 55.

2 Huguley, C M, et al, Cancer, 1975, 36, 1227

${ }^{3}$ Rimm, A A, and Bortin, M, Biomedicine, 1978, 28, 60.

+ Dudley, H, British Medical fournal, 1978, 1, 1344.

Gehan, E A, Biomedicine, 1978, 28, 13.

\section{Drug administration and food}

Most drugs are given by mouth-a route that is convenient and usually safe for the patient and makes no demands on medical time. Nevertheless, the amount of the drug that reaches the systemic circulation unchanged after oral intake is variable. In contrast with the certainty after an intravenous injection, this variable fraction is referred to as absolute bioavailability and depends on many factors. ${ }^{1}$

The formulation of the drug determines the rates of both disintegration and dissolution. Physicochemical characteristics are also important and include the crystalline structure of the preparation and the extent of ionisation of the drug molecule at the $\mathrm{pH}$ of the intestine. Highly ionised drugs such as the quaternary ammonium compounds are poorly and unreliably absorbed because they cannot cross cell membranes by passive diffusion. In contrast, non-ionised drugs which readily dissolve in lipid-propranolol, for example-may be almost completely absorbed from the gut. ${ }^{2}$ Gastric motility determines the rate of delivery of the drug to the small intestine. If the medicine is in solution by the time it reaches the small bowel absorption will be rapid and may be complete. In contrast, if gastric emptying is rapid and the drug dissolves comparatively slowly, rapid gastrointestinal transit may reduce bioavailability. Finally, bioavailability may be lowered by acid hydrolysis of the drug (for example, benzyl penicillin) or by its degradation by intestinal or hepatic enzymes. Biotransformation by such enzymes during absorption is often referred to as "metabolism at the first-pass."

Despite recent improvements in the information provided about drugs, comparatively few data sheets say whether the product should be taken with food or on an empty stomachthough food may have any one of several effects on the processes which determine bioavailability. Most studies have been based on single doses of drugs, and we need more work on patients taking regular medication. Nevertheless, the range of drugs whose bioavailability is improved by concurrent administration with food has been reviewed recently by Melander ${ }^{3}$; it includes the hypotensives hydrallazine, hydrochlorothiazide, and canrenone (the major metabolite of spironolactone) and possibly also the $\beta$-adrenoceptor antagonists propranolol and metoprolol. In contrast, quaternary ammonium compounds are less reliably absorbed in the presence of food, and this applies also to many antibiotics, particularly tetracyclines, ampicillin (but not amoxycillin), and the antituberculous drugs isoniazid and rifampicin. On the other hand, the absorption of nitrofurantoin is improved by concurrent administration with food.

How is absorption of drugs improved when they are given with meals? Among the possible explanations are alterations in tablet disintegration and drug dissolution and the variable effects of different types of meals on transit times through the gastrointestinal tract. Possibly, too, substances present in the food may affect the activity of enzymes located in the gut mucosa and liver. For inhibitors such actions would be immediate, but for other substances which induce microsomal enzyme oxidation the effect is likely to be seen only during regular exposure. One ingenious explanation recently proposed for the reported improved bioavailability of propranolol and metoprolol when given with food ${ }^{4}$ is that the stimulus of a meal might increase splanchnic blood flow and so improve the bioavailability of drugs (like propranolol) which undergo extensive metabolism at the first-pass through the liver. ${ }^{5}$ Using a computer model McLean et al $l^{5}$ produced findings consistent with this hypothesis-but all computer simulations are as good only as the programme used and the data supplied. Certainly the extraction of propranolol across the liver is dependent on blood flow, ${ }^{6}$ and hepatic blood flow is a major determinant of the half life of the drug when given intravenously. Nevertheless, the changes in the extraction ratio are relatively small with quite large variations in the hepatic blood flow. Furthermore, Wilkinson and Shand ${ }^{7}$ have shown that the bioavailability of propranolol given by mouth is chiefly related to the activity of microsomal drug-oxidising enzymes in the liver. While the bioavailability of drugs might be affected by changes in splanchnic blood flow after food, their proposal must await confirmation by careful studies.

Clearly we need to know more about drug administration and food, particularly during regular dosing. Food, its components and contaminants, probably has both short-term and long-term effects on absorption and biotransformation. The net effect on drug bioavailability cannot be predicted but must be measured in direct clinical studies of the drugs and foods in question. Furthermore, we may need to look closer at our current practice of studying drug bioavailability in patients and normal volunteers when they are fasting, for these may not represent the way in which the drug will be used in clinical practice. Perhaps, therefore, we should do our pharmacokinetic studies on new drugs under both fasting and nonfasting conditions using standardised test meals.

${ }^{1}$ George, C F, British Medical fournal, 1976, 2, 742.

2 Paterson, J W, European fournal of Clinical Pharmacology, 1970, 2, 127.

${ }^{3}$ Melander, A, Clinical Pharmacokinetics, 1978, 3, 337.

${ }^{4}$ Melander, A, Clinical Pharmacology and Therapeutics, 1977, 22, 108.

${ }^{5}$ McLean, A J, et al, Clinical Pharmacology and Therapeutics, 1978, 24, 5.

6 Weiss, Y A, et al, British fournal of Clinical Pharmacology, 1978, 5, 457.

7 Wilkinson, G R, and Shand, G D, Clinical Pharmacology and Therapeutics, $1975,18,377$.

\section{Fifty years of gold in rheumatoid arthritis}

Fifty years of treatment with gold in rheumatoid arthritis have elapsed-an anniversary that has been almost unnoticed. Jacques Forestier first used gold salts in rheumatoid arthritis in 1928 on an empirical basis, as they had appeared to have some effect on tuberculosis. ${ }^{1}$ Since then the popularity of gold has waned and waxed several times, but the amount used in Britain has increased steadily ever since the Empire Rheumatism Council report in 1961 finally established the clinical benefit of gold in a long-term controlled trial. ${ }^{2}$ It remains the last of the heavy metals in common clinical use, though we know little more about the reason for its effectiveness now than 50 years ago.

Nowadays rheumatoid arthritis is carefully defined, and 